\title{
Artelogie
}

Recherche sur les arts, le patrimoine et la littérature de I'Amérique latine

16 | 2021

Fotografía y migraciones, siglos XIX-XXI.

\section{El Taller de Creatividad: un dispositivo para la transformación personal}

\section{Guadalupe de la Cruz Aguilar Salmerón}

\section{OpenEdition}

\section{Journals}

Edición electrónica

URL: https://journals.openedition.org/artelogie/9512

DOI: 10.4000/artelogie.9512

ISSN: 2115-6395

Editor

Association ESCAL

Referencia electrónica

Guadalupe de la Cruz Aguilar Salmerón, «El Taller de Creatividad: un dispositivo para la transformación personal», Artelogie [En línea], 16 | 2021, Publicado el 28 enero 2021, consultado el 03 septiembre 2021. URL: http://journals.openedition.org/artelogie/9512 ; DOI: https://doi.org/10.4000/ artelogie.9512

Este documento fue generado automáticamente el 3 septiembre 2021.

Association ESCAL 


\title{
El Taller de Creatividad: un dispositivo para la transformación personal
}

\author{
Guadalupe de la Cruz Aguilar Salmerón
}

\section{Introducción}

1 El presente texto tiene su origen en la tesis doctoral: Procesos creativos y transformaciones subjetivas. La resigificación de la identidad a través de la experiencia artística dirigida, de Aguilar (2016), donde la investigación de los procesos creativos en el arte desde los paradigmas psicoanalítico y humanístico, los estudios de la cultura simbólica, algunos estudios del arte moderno y contemporáneo, así como la educación artística, dieron cuenta sobre la posibilidad de resignificar la experiencia vital por mediación de la práctica artística guiada.

De esta manera, se construyó un modelo de Taller de Creatividad, con la finalidad de proponer un espacio libre y seguro, propio para la reflexión personal, para crear conciencia y promover cambios significativos en la postura subjetiva de los participantes a favor del bienestar, a partir del planteamiento de distintos objetivos y de la exploración de diferentes lenguajes artísticos como el collage, el dibujo, la pintura, la escultura en barro, el performance, la música, la instalación, la escritura, la danza, entre otros:

El Taller de Creatividad [...] consistió en la creación de un espacio de reflexión personal en la interacción grupal, donde a partir del objetivo de cada una de las sesiones, se condujo al sujeto a una crisis definida como estado de reflexión, como antesala del cambio en la posición subjetiva, para la aparición de nuevas asociaciones a través de la experiencia artística [...], definido como sublimación, en nuestros términos: proceso creativo; esto significa romper los ciclos, las formas rígidas y repetidas [...] para promover en el sujeto la exploración, el conocimiento que lo lleven a resignificar la experiencia mediante la realización del deseo, a la toma de conciencia personal y 
situarlo en otro nivel de conciencia, que le permita definir algunos límites, asociar ciertos fragmentos para la reconstrucción de sí, para alcanzar mayor libertad interior. (AGUILAR, 2016: p 208)

4 Este modelo se ha implementado en numerosas ocasiones en instituciones y organizaciones públicas y privadas, con grupos de niños de 6 a 12 años, adolescentes de 12 a 16 y de 18 a 25 , así como adultos de 25 años en adelante. El modelo del Taller de Creatividad es una estructura diseñada para atender los procesos somáticos, psíquicos y simbólicos en relación, como aspectos universales de la naturaleza humana orientados al desarrollo de la consciencia personal, por lo que no están condicionadas por la edad, aunque para la realización de cada taller se toman en cuenta las necesidades y procesos específicos de cada periodo vital. En este sentido, el Taller de Creatividad expuesto en este texto, consta de diez sesiones de dos horas cada una.

5 Además de la observación de los resultados esperados durante la práctica artística guiada, el ordenamiento metodológico de la sesiones tiene una lógica deductiva, que se estableció a partir del desarrollo de los contenidos, estos tuvieron como hilo conductor las tres dimensiones de lo humano, que también se plantearon en términos de dimensiones de la consciencia, es decir, los planos físico, psíquico y espiritual. La dimensión física, se refiere a los aspectos de la corporalidad, la dimensión psíquica, corresponde a donde se asientan la dinámica de los afectos y las formas de pensamiento y la dimensión espiritual o simbólica corresponde a la articulación de las fases anteriores donde adquieren significado y trascendencia.

6 Esto se fundamentó en el paradigma psicoanalítico, específicamente en los aspectos que configuran la identidad en Freud (1923) en El yo y el ello, la cual se constituye personalidad a partir de la relaciones entre el yo, el ello y el superyó: "[...] la esencia del alma en un ello, un yo y un superyó." (FREUD, 1923: 9). Así como en Lacan (1954a) en La tópica de lo imaginario, sobre los tres registros de lo imaginario: real, imaginario y simbólico, en donde se encontraron elementos comunes y que además sostienen un correlato con el fenómeno de sublimación, como proceso simbólico o de transformación y que en este texto está planteado como proceso creativo, con el objetivo de analizar, explicar, poner en marcha y acompañar dichos procesos. Por lo anterior, el diseño del Taller de Creatividad se expone en el siguiente orden:

7 Empezamos con la primera sesión, que se ha definido como Introducción a la creatividad, donde se reflexiona sobre los bloqueos de la creatividad y el desarrollo del potencial creativo de cada participante. Con base en los conceptos de Waisburd (2003) en Creatividad y transformación y de Robles (2008) en Manual de grupo de crecimiento, por medio de un Dibujo y un Collage.

8 En la sesión dos se trabaja con El Yo cuerpo como principio de realidad, al explorar sus posibilidades y límites con supuestos de Freud (1923) en El yo y el ello y Bertherat (2002) en El cuerpo tiene sus razones, por medio de una actividad lúdica y el trazo de una Impronta corporal.

Durante la sesión tres se cuestionan las heridas de la vida y la posibilidad de su Autocuración con conceptos de Freud (1923) en El yo y el ello, de Bertherat (2002) en El cuerpo tiene sus razones y de Robles (2005) en Manual de grupo de crecimiento, a través de un Collage con material de primeros auxilios, sobre la impronta que trabajaron la sesión anterior. 
10 En la sesión cuatro se reflexiona sobre la Responsabilidad del autocuidado y el bienestar y se realizan ejercicios de Anti-gimnasia; con Foucault (2010) en Historia de la sexualidad y con Bertherat (2002) en El cuerpo tiene sus razones, respectivamente, a través de la exploración de sensaciones con diversos objetos y la elaboración de una escultura en Barro.

11 En la quinta sesión se realiza una introducción al Trabajo con las emociones para su reconocimiento y gestión, a través de la creación de un mandala sobre la gráfica Estirando el autoconcepto de Zinker (1997) en El proceso creativo en la terapia gestáltica y de Robles (2005) en Manual de grupo de crecimiento.

12 En la sexta sesión, Fluir bailando y pintando los participantes exploran el espacio con Telas, después expresan su experiencia en una Pintura, con base en los conceptos de Freud (1923) en El yo y el ello, de Waisburd, G. y Sefchovich, G. (1999) en Expresión corporal y creatividad y de Zinker (1997) en El proceso creativo en la terapia gestáltica.

13 Durante la sesión siete, El sujeto creador, las actividades se desarrollan en torno al Bodyart y Performance, donde los participantes crean el rol de sí mismos por medio de un disfraz, con base en los supuestos de Robles (2005) en Manual de grupo de crecimiento y Waisburd, G. y Sefchovich, G. (1999) en Expresión corporal y creatividad.

La sesión ocho consiste en plantear una dinámica de Creatividad a partir del caos, con objetos de desecho industrial, para resignificar qué don se puede aportar al mundo construyendo y pintando Esculturas de intervención, con base en algunas aportaciones de Bertherat (2002) en El cuerpo tiene sus razones así como de Waisburd, G. y Sefchovich, G. (1999) en Expresión plástica y creatividad.

En la sesión nueve el objetivo se dirige a la importancia de formar Conciencia de grupo a través de las reflexiones de Beuys (1985) sobre Un discurso sobre mi país en Joseph Beuys, de Bernárdez Sanchís (2003) por medio de la creación de Pinturas de gran formato en equipos.

16 El Taller de Creatividad concluye con la décima sesión a través de un Ritual de cierre, basado en los supuestos de Imber-Black, E., Roberts, J. y Whiting, R. (1997) en Rituales terapéuticos y ritos en la familia, para elaborar los aprendizajes y montar una exposición, a manera de galería de arte, con todos los trabajos de los participantes, para que sea visitada por sus familiares e invitados especiales.

17 Finalmente, la investigación concluyó que los procesos de formación de la identidad están profundamente ligados a los procesos creativos, pues los sujetos nos encontramos en un permanente proceso de cambio. En este sentido se vuelve pertinente preguntar si ¿El Taller de Creatividad promueve la reflexión y el cambio en la postura subjetiva del sujeto a favor de una experiencia vital más satisfactoria?

\section{Desarrollo}

Es entonces que a partir de la investigación doctoral se llegó a la creación de un concepto de proceso creativo construido desde una perspectiva psicoanalítica, el cual se ha publicado tanto en la tesis doctoral de Aguilar (2016), como en distintos textos de la misma autora, con las especificaciones relativas a cada objeto de estudio. Este, es fundamental para comprender el objetivo general y el trabajo que se realiza en el Taller de Creatividad, pues al ser un proceso complejo, también tiene la función de analizar, explicar, comprender, difundir y poner en marcha procesos para el aprendizaje significativo, en donde se encuentran interrelacionados procesos humanos y artísticos. 
A continuación se expondrá sólo a manera de síntesis, para no desviar la atención del objetivo del texto.

El proceso creativo, parte del funcionamiento del aparato psíquico y la dinámica pulsional que podemos definir en términos de energía, la cual se moviliza a partir de la aparición de una crisis, la cual es orientada hacia el placer para la satisfacción de la necesidad, la resolución del conflicto y la realización del deseo o en el caso contrario al displacer, donde no se elabora como tal: "[...] la actividad del aparato psíquico, aun del más desarrollado, está sometida al principio del placer, es decir, es regulada de manera automática por sensaciones de la serie placer-displacer [...]" (FREUD, 1915: p. 29). A esta energía que se desplaza, Freud (1915) le llamó pulsión y aunque en un origen la clasificó dentro de la teoría sexual, también la diferenció y la circunscribió a una forma de energía desexualizada y sublimada manejada por el yo. Por otro lado, la pulsión, posee una doble naturaleza, una del orden de lo psíquico y otra de lo somático, es decir, acontece en ambas realidades, lo que establece relaciones de muy diversa índole: "La «pulsión» nos aparece como un concepto fronterizo entre lo anímico y lo somático, como un representante \{Repräsentant\} psíquico de los estímulos que provienen del interior del cuerpo y alcanzan el alma." (FREUD, 1915: p. 29). Y aunque la traducción francesa también suele llamarle instinto o tendencia, para Laplanche (2002), la pulsión es una especie de empuje o estímulo que detona un acto: "[...] lo que pone en marcha." (LAPLANCHE, 2002: p. 34) Si bien se puede afirmar que la pulsión tiene la función de resolver una necesidad, esta no siempre se moviliza por la vía adecuada, no obstante, en el proceso creativo, esta sólo cumple su objetivo mediante su destino como fenómeno de sublimación.

Para comprender este fenómeno, es importante saber que la pulsión está compuesta por cuatro fases: fuente, esfuerzo, meta y objeto y que sus interrelaciones la orientan hacia diferentes fines, siendo el que se expone a continuación el que caracteriza al proceso creativo o sublimación.

21 Para Freud (1915), la fuente es el origen de la pulsión, es el lugar psíquico o somático de donde surge, aunque hay que tomar en cuenta que sea cual fuere su origen, uno influye al otro y se encuentran en una relación permanente: "Por fuente \{Quelle\} de la pulsión se entiende aquel proceso somático, interior a un órgano o a una parte del cuerpo, cuyo estímulo es representado \{repräsentiert\} en la vida anímica por la pulsión." (Freud, 1915: 29) Para Laplanche (2002) la fuente, no sólo es el origen de la pulsión sino que puede ser incluso cualquier acción o proceso: "[...] cualquier proceso somático, aun cualquier modificación difusa, cualquier acción - incluso psíquica -[...]." (LAPLANCHE, 2002: p. 36)

Para Freud (1915), el esfuerzo es la cantidad de energía que necesita la pulsión para ser consumada: "Por esfuerzo \{Drang\} de una pulsión se entiende su factor motor, la suma de fuerza o la medida de la exigencia de trabajo que ella representa \{repräsentieren\}." (FREUD, 1915: p. 29). Por su parte, para Laplanche (2002) el esfuerzo es de hecho la pulsión: “[...] lo que <<impulsa >> a una acción." (LAPLANCHE, 2002: p. 38) La meta es en sí misma la satisfacción de la pulsión: "La meta \{Ziel\} de una pulsión es en todos los casos la satisfacción que sólo puede alcanzarse cancelando el estado de estimulación en la fuente de la pulsión." (FREUD, 1915: p. 29) Que incluso, puede quedar parcialmente satisfecha o como alguna satisfacción desviada. Según Laplanche, (2002) la meta se realiza por la acción, es verbo: “[...] es la acción.” (LAPLANCHE, 1915: p. 35). En este sentido, y para el interés del texto, se trata de una acción guiada que imagina, da forma y se concreta en el objeto artístico. 
24 Finalmente el objeto es el lugar donde se realiza la pulsión: "El objeto \{Objekt\} de la pulsión es aquello en o por lo cual puede alcanzar su meta." (FREUD, 1915: p. 29). Para Laplanche y Pontalis (2004), el objeto puede ser psíquico o somático, exterior o interior, intangible o materializado, es decir, el elemento más variable de los que conforman la pulsión, siempre es sustantivo: “[...] es aquello en lo cual y mediante lo cual la pulsión busca alcanzar su fin, esto es, cierto tipo de satisfacción. Puede tratarse de una persona o de un objeto parcial, de un objeto real o de un objeto fantaseado." (LAPLANCHE, J. Y PONTALIS, J, 2004: p. 258). En su caso, para Lacan (1954) el objeto es: [...] un punto de fijación imaginaria que da, bajo el registro que esté, satisfacción a una pulsión." (LACAN, 1960: p.61), En dichos términos y siguiendo el hilo conductor de este escrito, se trata del objeto artístico ya creado, que puede ser un collage, un dibujo, una pintura, una escultura, un performance, un escrito, etc.

Asimismo, para complementar el concepto de proceso creativo, se abordaron los tres registros psíquicos de Lacan (1954a) en La tópica de lo imaginario y sus relaciones, donde se encontró que funcionan como fases que ilustran el proceso de configuración de la identidad, así como cualquier proceso de transformación y que podemos establecer como condición simbólica. Estos tres registros son: el real, lo imaginario y lo simbólico, donde lo real es el primer encuentro con la realidad, tal como se presenta, es decir, lo no conocido, no estructurado y que necesita ser elaborado, es: "[...] el caos original [...]" (LACAN, 1954a: p. 33) y que para configurar dicho proceso, lo liga con lo imaginario, en donde convergen y dialogan opuestos, es este caso elementos de la conciencia y del inconsciente, pensamientos y afectos y que define como: “[...] el nacimiento del yo [...]"(LACAN, 1954a: p. 33), finalmente, estos opuestos que se resuelven en la imaginación mediante una síntesis, posicionan al sujeto de cara a eso asumido, comprendido, significativo, simbólico y que distingue como: "[...] las posiciones del sujeto." (LACAN, 1954a: p. 33). Por lo que se puede observar que al producirse una condición simbólica para el sujeto ocurre la transformación de la subjetividad.

En este sentido, afirma Lacan (1954b) en El universo simbólico, que llevar al sujeto al orden simbólico, construye crear un universo completo, pues al ser alcanzada la condición simbólica, la realidad es percibida como una totalidad:

[...] el orden simbólico, por cuanto se plantea siempre como un todo, como algo que forma por sí sólo un universo-y que incluso constituye el universo como tal, en tanto que distinto del mundo-, debe estar igualmente estructurado como un todo, vale decir que forma una estructura dialéctica acabada, completa. (LACAN, 1954b: p. 14)

Finalmente, es importante comprender que todo proceso creativo es un procesamiento simbólico, pues ambos se componen por las mismas fases y los definen las mismas pautas. Por lo anterior, se presenta una especie de fórmula que clarifica dichos procesos y es al mismo tiempo una metodología para diseñar el proceso creativo:

$$
\text { (síntesis) - Proceso creativo (como mecanismo constructor de transformación) }
$$

Real + Imaginario (Relaciones entre la conciencia y el inconsciente - pensamiento y afectos) $=$ Simbólico - Proceso creativo

Es entonces que, teniendo como elemento base las conceptualizaciones anteriores, se plantea a continuación la estructura del Taller de Creatividad, en donde en cada sesión 
tiene por objetivo llevar a los sujetos a la condición simbólica, a través de la práctica artística guiada.

\section{Primera sesión: Introducción al proceso creativo}

31 La primera sesión, ha sido llamada: Introducción al proceso creativo, donde se establece un clima seguro para introducir a los participantes a los procesos de creación, teniendo como fundamento la idea que todos somos creativos, con base en conceptos de Waisburd (2003) en Creatividad y transformación, Robles (2008) en Manual de grupo de crecimiento. El objetivo es desarticular las falsas creencias respecto de la creatividad artística y reflexionar sobre sus bloqueadores y la recuperación creativa, donde se propone la realización de un dibujo que representa los bloqueos y un collage como símbolo de dicha recuperación.

La sesión inicia con la Inducción, la cual corresponde a la fase de entrada, en donde se recibe a los participantes y se les facilita realizar la transición del afuera hacia el interior del espacio.

La Introducción tiene lugar una vez que los participantes disponen la atención en el salón, entonces se plantea el objetivo de la sesión, la reflexión general y se dan las orientaciones necesarias para el trabajo. El tema sobre la Introducción al proceso creativo se ha abordado a partir de reflexionar sobre el contexto cultural, el cual le ha dado gran relevancia al desarrollo cognoscitivo y se ha dejado de lado el desarrollo de la inteligencia emocional, que incluye la expresión artística, la comunicación eficaz, el desarrollo personal, entre otros. Para Teresa Robles (2008), la salud mental se basa en el equilibrio de ambos procesos de desarrollo, los cuales se encuentran en una constante comunicación y a los que liga con la teoría de los dos hemisferios cerebrales, esta les atribuye funciones distintas, de manera que mediante su interacción es posible alcanzar la máxima creatividad. Sabemos por Waisburd (2003) que la creatividad se ha estudiado desde diferentes modelos teóricos, la mayoría supone que el potencial creativo es una cualidad esencial del ser humano, sin embargo, es importante facilitar las condiciones para su libre desarrollo, ya que durante la vida se suelen sufrir experiencias que interfieren en la percepción y bloquean el proceso, lo que frecuentemente se convierte en actitudes negativas que impiden la expresión del sí mismo y limitan el desarrollo. De esta manera, los bloqueos condicionan los aspectos físicos, cognoscitivos, emocionales y espirituales de las personas, así, la energía como pulsión, busca un destino en la conciencia, si fluye y arriesga es creativa, lo cual puede producir cambios significativos. Por el contrario, si la energía se estanca, la conciencia se establece en la negación y el miedo. En este orden, propone las siguientes actitudes para recuperar la creatividad:

Recuperación creativa:

- Comprensión de nuestros bloqueos

- Análisis de nuestra formación creativa, qué favoreció o anestesió el proceso

- Integrar el cuerpo con la mente, la emoción y el espíritu

- Actitudes de amor, apertura, respeto, aceptación, humildad y libertad

- Conciencia del potencial creativo y del camino para desarrollarlo

- Crear experiencias y buscar ambientes que sensibilicen y despierten las percepciones

- Manifestar en un ambiente seguro emociones reprimidas para liberar la energía. La energía sigue a la conciencia 
- Experimentar y conocer el poder de la mente

- Desarrollo de la visualización, la meditación, la imaginación, la fantasía

$$
\begin{aligned}
& \text { guiada, en donde se recomienda reorganizar el espacio para } \\
& \text { encontraron y concluir con los comentarios sobre la experiencia. }
\end{aligned}
$$

\section{Segunda sesión: El Yo, principio de realidad. El cuerpo con sus posibilidades y límites} cuerpo, pues por su mediación se establecen las relaciones con la realidad y con los demás. Esto se ha fundamentado en algunos supuestos de Freud (1923) en El yo y el ello y con Bertherat (2002) en El cuerpo tiene sus razones. Por lo que la sesión tiene el objetivo de crear conciencia con respecto a los límites y el potencial adaptativo del cuerpo, para la construcción del auto concepto, a través de una actividad lúdica con globos y el trazo de una impronta corporal.

La sesión inicia con la Inducción, como la fase de entrada en donde se recibe a los participantes y se les facilita realizar la transición del afuera hacia el interior del espacio.

41 La Introducción tiene lugar una vez que los participantes centran la atención en el salón, se plantea el objetivo de la sesión, la reflexión general y se dan las orientaciones necesarias para el trabajo. El tema sobre: El Yo, principio de realidad, se ha abordado a partir de comprender lo que para Freud (1923) es el yo: el cuerpo, el cual se torna consciente de sí al experimentar la percepción, tanto en forma de sensaciones como de sentimientos: "El Yo es sobre todo una esencia-cuerpo." (FREUD, 1923: p. 2) Por su parte, para Bertherat (2002) ser consciente del cuerpo significa abrirse a la totalidad del ser, porque en él están representadas las dimensiones física, psíquica y espiritual, las cuales forman una unidad. Esta consciencia implica comprender que ocupa un lugar y un espacio, que se le puede medir y pesar, que se adapta y a la vez tiene limitaciones, que la percepción es el medio para conocer y relacionarse con el mundo; que es único e irrepetible; que es temporal y se rige por ciclos establecidos por su naturaleza, estos no pueden ser condicionados, pero tal puedan ser modificados, estos implican nacer, crecer, desarrollarse, reproducirse, deteriorarse y morir, asimismo se renueva, pues está sincronizado de manera automática y posee una información genética heredada que regula sus pautas fisiológicas; al desarrollarse se adapta y se conserva; tiene constantes necesidades por atender, como protección, vestido, alimento, cuidados de salud, contención, cariño, descanso, actividad física, entre otras; el cuerpo tiene memoria; también posee un lenguaje propio que manifiesta en formas de bienestar y malestar. Es nuestra única realidad concreta y representa todo lo que somos. De esta manera, nos relaciona con el mundo que nos rodea y con los demás. Al trabajar, hace 
posible materializar lo que sentimos y pensamos, lo que soñamos y deseamos, por lo que existir significa crearse constantemente. De acuerdo con Bertherat (2002), es importante tener las llaves de nuestro cuerpo, habitar nuestra casa, tomar posesión de él, pues el cuerpo es lo que somos. De otra manera, si se reprimen sensaciones o disminuimos nuestras dimensiones reales ante nosotros mismos, aparece la sensación de existir incompletos.

La fase de Desarrollo corresponde a la práctica artística guiada, la cual en esta sesión está planeada en dos partes, durante la primera los participantes realizan una exploración sobre la extensión de su cuerpo mediante el juego con globos, durante la segunda actividad se dibuja una impronta corporal, donde marcan sus propios límites e insertan en el interior símbolos sobre temas con los que se identifican, sobre un papel de gran formato.

Finalmente, la fase de Cierre de la sesión, procede cuando termina la práctica artística guiada, en donde se recomienda reorganizar el espacio para dejarlo como lo encontraron y concluir con los comentarios sobre la experiencia.

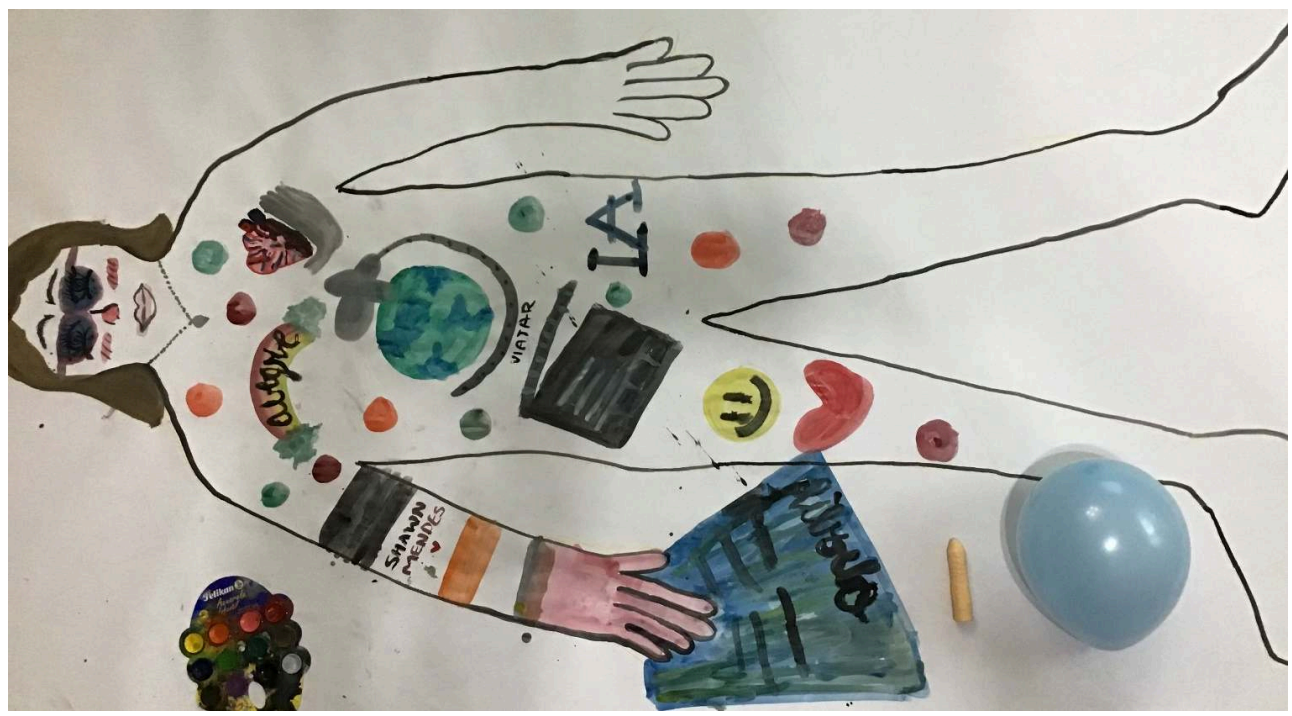

Imagen 1: Aguilar, G. 2020, Sesión 2 El Yo, principio de realidad

\section{Tercera sesión: Auto-curación}

Durante la tercera sesión, que se ha llamado Auto-curación, la atención está centrada en el cuerpo, con el objetivo de descubrir las heridas propias para curarlo, identificar sus necesidades, desarrollar la responsabilidad del autocuidado y explorar caminos de integración. Por lo que se trabaja con conceptos de Freud (1923) en El yo y el ello, de Bertherat (2002) en El cuerpo tiene sus razones y de Robles (2005) en Manual de grupo de crecimiento, mediante la creación de un collage con material de primeros auxilios sobre la impronta de la sesión anterior.

La sesión inicia con la Inducción, como fase de entrada, en donde se recibe a los participantes y se les facilita la transición del afuera hacia el interior del espacio.

La Introducción se realiza una vez que los participantes tienen la atención en el salón, se plantea el objetivo de la sesión, la reflexión general y se dan las orientaciones necesarias para el trabajo. El tema sobre Auto-curación tiene como punto de partida 
comprender con Freud (1923) que es posible hacer conscientes las percepciones sensoriales que vienen del exterior así como las percepciones interiores, llamadas sensaciones y sentimientos, respectivamente, esto es fundamental porque significa que cualquier estímulo exterior o interior, al establecer relaciones en ambos sentidos, generan registros somáticos y anímicos. En este orden, sabemos que en el transcurso de la vida aparecen situaciones que nos lastiman y nos hacen tomar distancia de nosotros mismos para evitar el dolor. De esta manera, se encontró que Teresa Robles (2005) trabaja la Auto-curación, teniendo como idea central la siguiente sugestión hipnótica: "[...] todas las heridas cicatrizan si las dejamos cicatrizar, si dejamos de meterles el dedo y arrancarles la costra [...]" (ROBLES, 2005: p. 36) De tal suerte que se puede afirmar con ella que:

Las heridas son [...] grandes o chicas, superficiales o profundas, antiguas o recientes [...] Las heridas necesitan [...] y cuando ya cicatrizaron, se vuelven recuerdos, ya sin dolor, de esos malos momentos que ya pasaron. (ROBLES, 2005: p. 36)

Por lo anterior, agrega Bertherat (2002), es necesario incorporar todos los sentidos de percepción para identificar sensaciones y necesidades, así como los males físicos para manejarnos con mayor libertad. Esto significa que la toma de consciencia corporal y emocional facilita la configuración de una imagen satisfactoria de nosotros mismos, la recuperación del sentido de individualidad, la gestión asertiva de las emociones, la iniciativa, la autoconfianza y la espontaneidad.

La fase de Desarrollo corresponde a la práctica artística guiada que consiste en trabajar las heridas sobre la impronta de la sesión pasada, aplicando material de primeros auxilios, a manera de collage.

Finalmente, la fase de Cierre de la sesión, procede cuando termina la práctica artística guiada, en donde se recomienda reorganizar el espacio para dejarlo como lo encontraron y concluir con los comentarios sobre la experiencia.

\section{Cuarta sesión: La responsabilidad del autocuidado y el bienestar}

50 La cuarta sesión: La responsabilidad del autocuidado y el bienestar, se fundamentó en el desarrollo de la percepción, con supuestos de Bertherat (2002) en El cuerpo tiene sus razones, así como la reflexión respecto al cuidado de sí, de Foucault (2010), en Historia de la sexualidad: La inquietud de sí, con el objetivo de crear conciencia sobre la responsabilidad que tenemos de procurarnos autocuidado, favorecer el desarrollo de la sensibilidad y el bienestar, mediante la exploración de objetos con distintas texturas, olores, sabores y el modelado de una escultura en barro, con los ojos tapados.

51 La sesión inicia con la Inducción, esta corresponde a la fase de entrada, en donde se recibe a los participantes y se les facilita realizar la transición del afuera hacia el interior del espacio.

52 La Introducción comienza una vez que los participantes tienen la atención en el salón, se plantea el objetivo de la sesión, la reflexión general y se dan las orientaciones necesarias para el trabajo. En el planteamiento del tema sobre La responsabilidad del autocuidado y el bienestar se está afirmando la importancia de desarrollar una 
consciencia razonada sobre el cuidado de sí y con relación al bienestar, se busca la realización del deseo promovida por la búsqueda del placer, con la finalidad de encontrar motivos que faciliten el disfrute de la vida. En este sentido, Bertherat (2002) afirma que habitar el cuerpo significa aprender a manejarlo con libertad, esto es, favorecer el desarrollo para vivir como personas completas, integradas, bien constituidas, auténticas y armónicas; porque vivir nuestra corporalidad de manera saludable implica conocer sus sensaciones, lo que resulta en saber organizar y manejar los movimientos desde el interior, aceptarnos y querernos como somos, pues cuando nos sentimos bien en el cuerpo podemos percibir, admitir y desarrollar sus sensaciones, conocer el mundo que nos rodea y tener la confianza de explorarlo, liberarnos de los condicionamientos que nos imponen los espacios exteriores y vivirnos con libertad. Además, es importante para conocer los límites propios, lo que causa daño y dolor, para entender los lenguajes del cuerpo y procurar su cuidado. Es por ello que se considera necesario despertar los cinco sentidos, pues como se menciona con anterioridad, conocer las sensaciones también nos permite conectarnos con lo que sentimos: "Sentirse bien en el cuerpo ¿no significa ante todo poder sentirse, admitir, percibir y desarrollar sus sensaciones?" (BERTHERAT, 2002: p. 64). Para Foucault (2010), el arte de la existencia reside en el principio de "[...] cuidar de uno mismo." (FOUCAULT, 2010: $p$. 50), pues afirma, que en este principio se centran las necesidades que le otorgamos, guían su desarrollo y ordenan su práctica. De acuerdo con Foucault (2010), se encuentran registros sobre la consciencia del autocuidado en occidente desde las civilizaciones helenística y romana, donde el sujeto posee la consciencia para el crecimiento personal, para valorar de manera privada su conducta y el interés hacia el desarrollo de su consciencia personal.

La fase de Desarrollo corresponde a la práctica artística guiada, la cual consiste en explorar con los ojos tapados una gran diversidad de objetos de distintas dimensiones, texturas, olores y sabores. La exploración concluye con la realización de una escultura en barro.

Finalmente, la fase de Cierre de la sesión, procede cuando termina la práctica artística guiada, en donde se recomienda reorganizar el espacio para dejarlo como lo encontraron y concluir con los comentarios sobre la experiencia. 


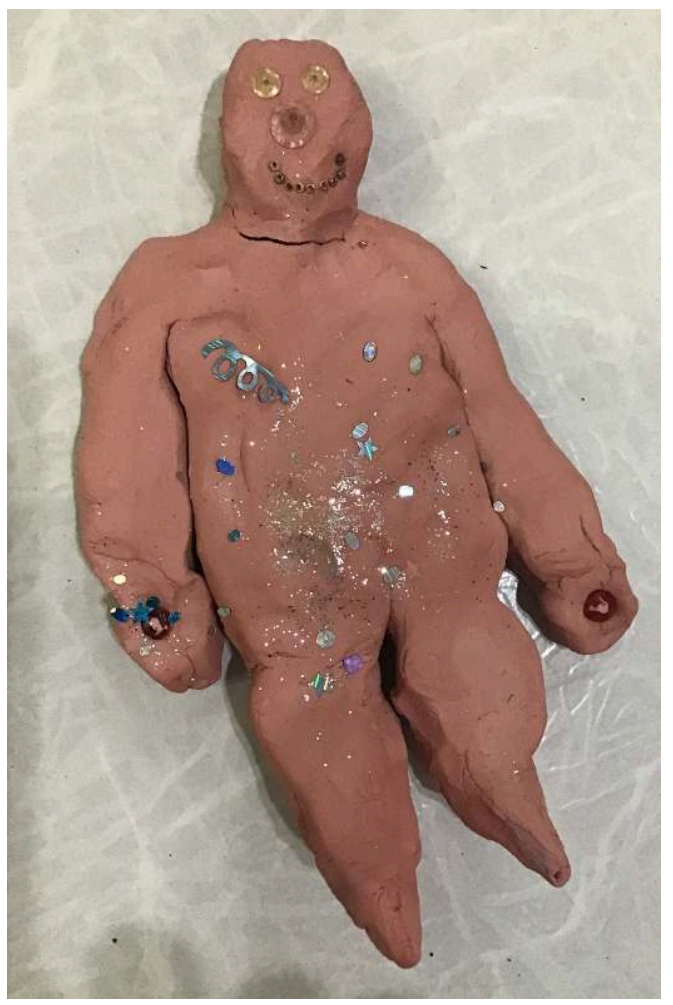

Imagen 2: Aguilar, G. 2020, Sesión 4 La responsabilidad del autocuidado y el bienestar

\section{Quinta sesión: El Trabajo con las emociones}

La quinta sesión El Trabajo con las emociones, está fundamentada en los supuestos referentes al concepto de pulsión, como elemento fronterizo entre lo somático y lo anímico y la estructura anímica de Freud (1915), (1923) en Pulsiones y destinos de pulsión y El yo y el ello respectivamente, en el trabajo sobre las emociones y su gestión de Robles (2005) en Manual de grupo de crecimiento y en la teoría sobre la polarización de las emociones, con la que es factible propiciar la integración a la personalidad, de Zinker (1997) en El proceso creativo en la terapia gestáltica, con el objetivo de conocer la función de las emociones y aprender la dinámica de su manejo, mediante la creación de un mandala.

La sesión tiene como punto de inicio la Inducción, esta corresponde a la fase de entrada, en donde se recibe a los participantes y se les facilita realizar la transición del afuera hacia el interior del espacio.

La Introducción empieza una vez que los participantes tienen la atención en el espacio, se plantea el objetivo de la sesión, la reflexión general y se dan las orientaciones necesarias para el trabajo. En el planteamiento del tema sobre El Trabajo con las emociones se fundamenta en la teoría de la estructura anímica de Freud (1923), la cual se encuentra constituida por un yo, un ello y un superyó y sus interrelaciones: "[...] la esencia del alma en un ello, un yo y un superyó." (FREUD, 1923: 9). Como sabemos, el yo de Freud (1923) es un yo cuerpo, el principio de realidad y representante de la razón, el cual tiene la función de ser la parte que organiza coherentemente los procesos anímicos del sujeto, donde nace la consciencia sobre la percepción interior y exterior, la cual aparece en forma de sensaciones y sentimientos, que provienen de una gran diversidad 
de niveles del aparato anímico y/o del mundo que lo rodea. Es en el yo, donde la percepción cumple la función que en el ello le corresponde a la pulsión, donde se origina la energía que contiene las pasiones: "El yo es el representante \{repräsentieren\} de lo que puede llamarse razón y prudencia, por oposición al ello, que contiene las pasiones." (FREUD, 1923: p. 5). En este orden, afirma Freud (1915), la pulsión pertenece a los ámbitos de lo anímico y de lo somático, se expresa a través del ello en el yo, se trata de la energía que se desplaza entre el alma y el cuerpo, sensibilizándolos. Además, existe un tercer elemento que regula la energía pulsional en el aparato anímico, el superyó, este opera como consciencia moral, facilitando o impidiendo la expresión de dicha energía. Se trata entonces que esta energía en desplazamiento es en sí la dinámica de las emociones, las cuales en un origen se presentan como el lenguaje del inconsciente y es en consciencia donde se les puede gestionar; su función consiste en manifestar lo que sucede en nuestro interior con relación a la experiencia exterior. De esta manera, no controlamos lo que sentimos, pero si somos responsables de cómo lo expresamos. Si aprendemos a conocer nuestras emociones y darles nombre, tendremos una mejor comprensión de nosotros mismos, de nuestra realidad y desarrollaremos la capacidad de ser asertivos en su manejo. De acuerdo con Robles (2005), es importante reconocer nuestras emociones, porque somos lo que sentimos y lo que pensamos, lo que deseamos y también nuestro cuerpo. Además, hemos aprendido a dividir el mundo en pares opuestos, en lugar de mitades complementarias o totalidades. En este orden, la teoría de las polaridades de Zinker (1997) propone que estas mismas emociones en oposición forman una unidad y que al comprenderlas como tales, facilitan la integración de la personalidad:

Mi teoría sobre las polaridades sostiene que si no me permito ser malvado, nunca seré genuinamente bondadoso. Si estoy en contacto con mi propia maldad y amplío esa parte de mí mismo, mi bondad, cuando se manifieste, será más rica, más plena, más completa. Si no me permito a mí mismo tener contacto con mi femineidad, mi masculinidad será exagerada, hasta perversa [...]. (ZINKER, 1997: p. 162)

De tal suerte que podemos confirmar que las emociones siempre tienen la función de enseñarnos cómo nos estamos relacionando con lo que sucede, resolverlas implica siempre un trabajo personal.

La fase de Desarrollo corresponde a la práctica artística guiada, la cual consiste en pintar un mandala, situando en los extremos opuestos de toda la circunferencia los nombres de emociones complementarias y se distinguen pintando cada emoción con un color diferente.

61 Finalmente, la fase de Cierre de la sesión, procede cuando termina la práctica artística guiada, en donde se recomienda reorganizar el espacio para dejarlo como lo encontraron y concluir con los comentarios sobre la experiencia.

\section{Sexta sesión: Fluir bailando y pintando}

62 La sexta sesión Fluir bailando y pintando está sustentada en los principios de Freud (1923) en El yo y el ello, relativos al cuerpo, a sus sensaciones y sentimientos, así como de Waisburd, G. y Sefchovich, G. (1999) en Expresión corporal y creatividad, quienes proponen una serie de ejercicios para la facilitación de procesos creativos por medio de la expresión corporal y que Zinker (1997) en El proceso creativo en la terapia gestáltica, complementa subrayando a la práctica artística como un acto de valentía. La sesión 
tiene el propósito de facilitar el fortalecimiento del yo, a través de la libre expresión tanto corporal como plástica, con telas y la creación de una pintura.

La sesión inicia con la Inducción, como fase de entrada, en donde se recibe a los participantes y se les facilita realizar la transición del afuera hacia el interior del espacio.

La Introducción empieza una vez que los participantes tienen la atención en el salón, se plantea el objetivo de la sesión, la reflexión general y se dan las orientaciones necesarias para el trabajo. La exposición del tema Fluir bailando y pintando, está basada en la propuesta de Freud (1923), relativa al yo cuerpo, no sólo como superficie sino como proyección de una superficie, donde se originan las percepciones a partir de la experiencia. Estas percepciones son las que se identifican en la categoría del placerdisplacer, las cuales al promover el movimiento libre del cuerpo, facilitan el tránsito del displacer al placer. De acuerdo con Waisburd, G. y Sefchovich, G. (1999), la expresión corporal tiene por objetivo la creación de procesos creativos a través de la libre expresión y la comunicación, lo que permite aprender las posibilidades del cuerpo en movimiento, el manejo del espacio y del material, pues significa rebasar los propios límites y atreverse a salir de los moldes establecidos, esto fortalece la autoconfianza y crea la libertad de plantarse de frente a la realidad y ante los demás, de tal suerte que afirmamos con Zinker (1997): la creatividad es un acto de valentía.

La fase de Desarrollo corresponde a la práctica artística guiada, la cual para esta sesión consiste en explorar el espacio con telas el tiempo que se considere necesario y finalizar pintando la experiencia.

Finalmente, la fase de Cierre de la sesión, procede cuando termina la práctica artística guiada, en donde se recomienda reorganizar el espacio para dejarlo como lo encontraron y concluir con los comentarios sobre la experiencia.

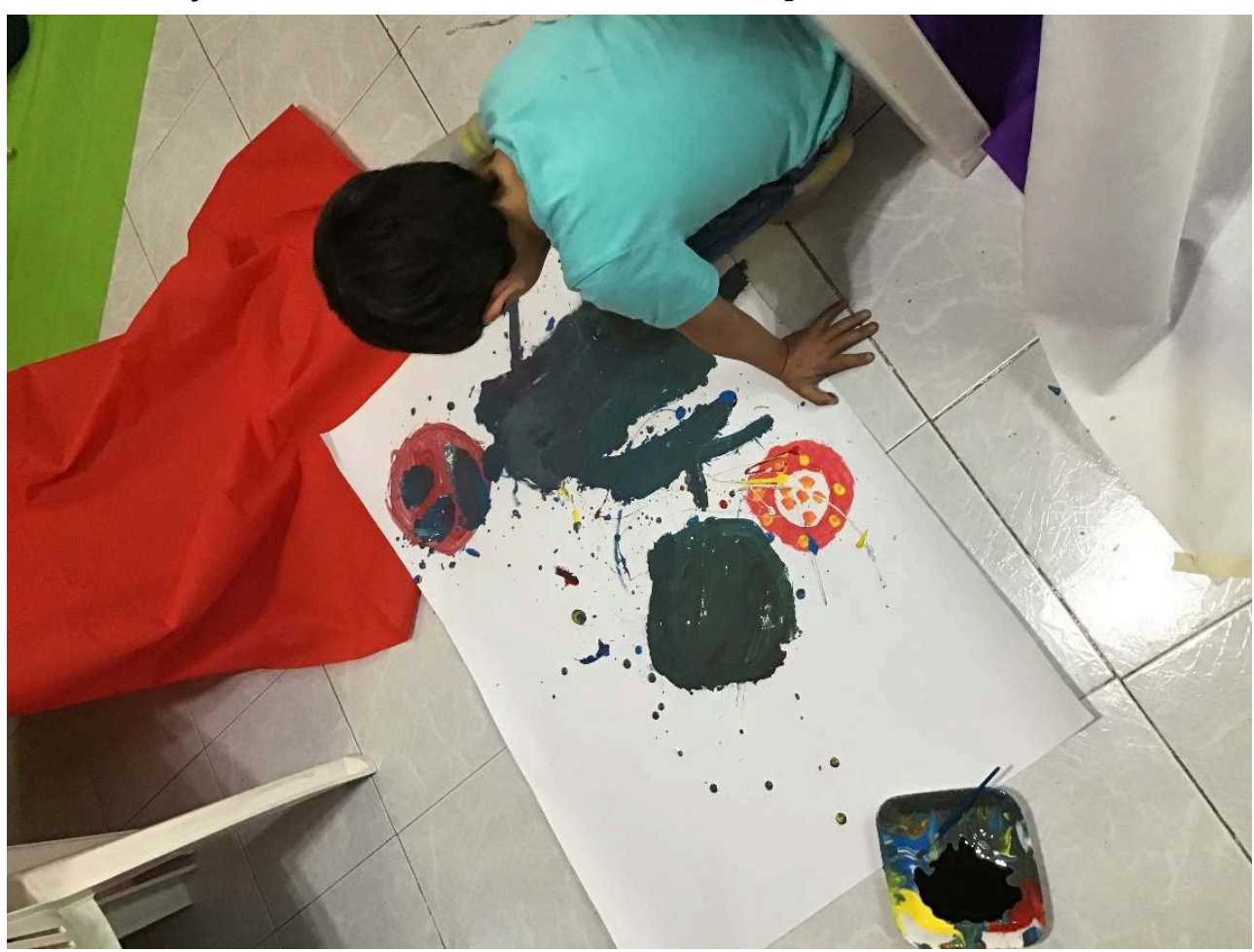

Imagen 3: Aguilar, G. 2020, Sesión 6 Fluir bailando y pintando 


\section{Séptima sesión: El sujeto creador}

$$
\begin{aligned}
& \text { permitan mirarla. El problema es que estén pegados y no nos los podamos quitar. El } \\
& \text { problema es que creamos que esos somos, que los confundamos con nuestra piel. }
\end{aligned}
$$
(ROBLES, 2005: p.57)

71 No obstante, también son necesarios para interactuar con el mundo que nos rodea, para representar nuestros roles y para protegernos. Lo importante es que sepamos distinguir que no somos nuestros disfraces, ni lo que parecemos ser, simplemente ser conscientes de ser lo que somos. Finalmente, se concluye con las reflexiones de Waisburd, G. y Sefchovich, G. (1999) quienes proponen al cuerpo como instrumento para transmitir emociones y sentimientos, como ente de razón, intuitivo, susceptible a la experiencia, al aprendizaje, al conocimiento, a la percepción y a la comunicación: "El cuerpo es el instrumento que nos permite participar activamente en la sinfonía de la vida." (WAISBURD, G Y SEFCHOVICH, G., 1999: p. 16) 
73 Finalmente, la fase de Cierre de la sesión, procede cuando termina la práctica artística guiada, en donde se recomienda reorganizar el espacio para dejarlo como lo encontraron y concluir con los comentarios sobre la experiencia.

\section{Octava sesión: Creatividad a partir del caos}

La octava sesión Creatividad a partir del caos, se basó en el caos como principio creador. Esto, con el objetivo de reflexionar sobre la integración de aquellas partes nuestras que hemos dejado afuera, para constituirnos como personas más armónicas; así también para atender la necesidad de dar forma a la aparente confusión en la que actualmente vivimos. En donde se integraron conceptos de Bertherat (2002) en El cuerpo tiene sus razones, relativos a la experiencia sensorial, mediante la elaboración de esculturas de intervención, ofrecidas al mundo como un regalo del sí mismo, construidas con material de deshecho industrial.

La sesión inicia con la Inducción, la cual corresponde a la fase de entrada, en donde se recibe a los participantes y se les facilita realizar la transición del afuera hacia el interior del espacio.

76 La Introducción inicia una vez que los participantes tienen la atención en el salón, se plantea el objetivo de la sesión, la reflexión general y se dan las orientaciones necesarias para el trabajo. De esta manera, la exposición de la sesión Creatividad a partir del caos, se basa en orientar la atención sobre todo aquello que forma parte de nosotros y que no hemos integrado, es decir, lo que se ha reprimido, olvidado, perdido o causa miedo, vergüenza o culpa o, simplemente se encuentra fuera del margen de valoración. En este sentido, se crea una crisis que impacta al participante por la disposición desordenada de los objetos, pues la transgresión del espacio traspasa los límites de lo visible, con la intención de generar un proceso creativo donde retome y dé forma a lo olvidado, lo roto, al reconstruir, reconfigurar y resignificar. Para que el sujeto amplíe la comprensión de su capacidad creativa que lo conduce a asumir dicho caos desde la propia autonomía, al orientar su voluntad para funcionar al máximo y expresar armonía, a través de sus decisiones y acciones, esto significa alcanzar la condición simbólica: "[...] descubrirse, no desde el exterior, sino desde el interior de sí mismo." (BERTHERAT, 2002: p. 25). Lo que supone, dicen Waisburd, G. y Sefchovich, G. (1999), adquirir aprendizajes significativos pues modifican hábitos y conductas.

77 La fase de Desarrollo corresponde a la práctica artística guiada, la cual consiste en construir y pintar un objeto artístico para ofrecerlo como un don de sí mismo al mundo a manera de instalación, realizada con desechos industriales como cajas, frascos, botes de metal y plástico, todo tipo de envases y embalajes.

78 Finalmente, la fase de Cierre de la sesión, procede cuando termina la práctica artística guiada, en donde se recomienda reorganizar el espacio para dejarlo como lo encontraron y concluir con los comentarios sobre la experiencia. 


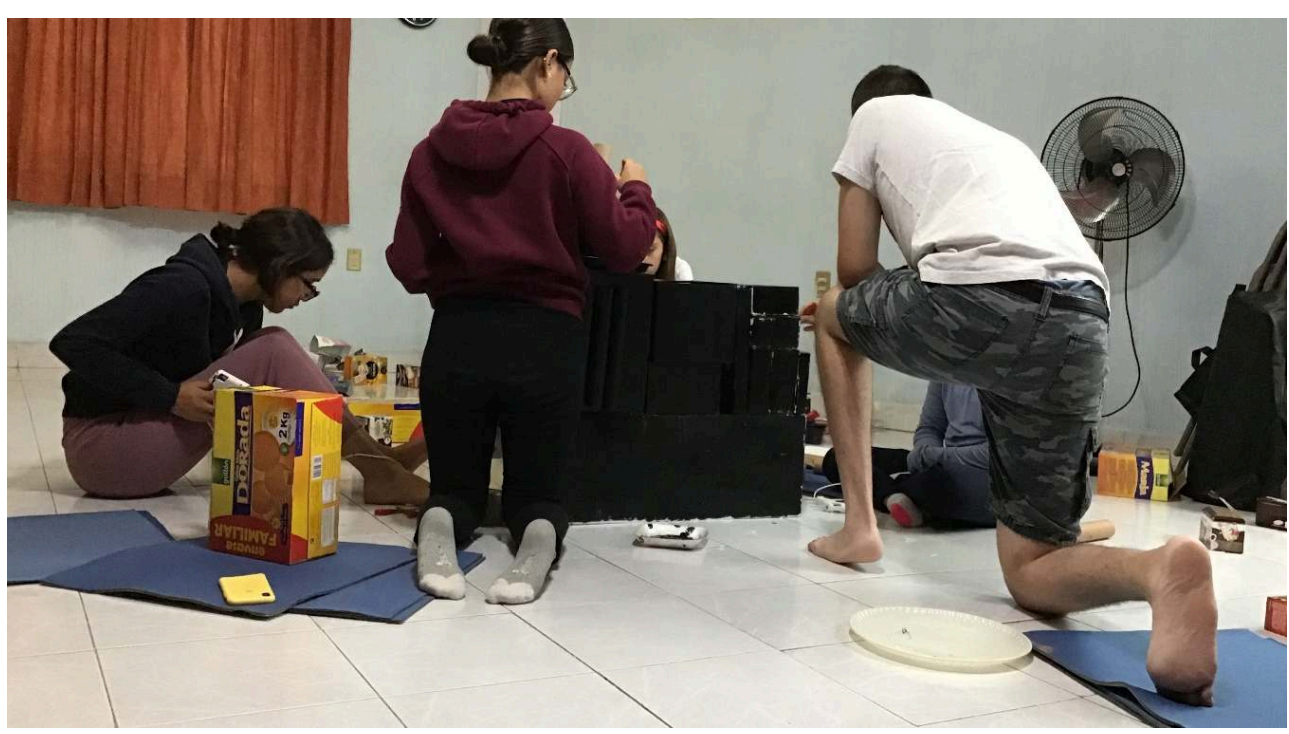

Imagen 4: Aguilar, G. 2020, Sesión 8 Creatividad a partir del caos

\section{Novena sesión: La conciencia de grupo}

La novena sesión La conciencia de grupo se fundamenta la propuesta de Joseph Beuys (1958) con respecto a Un discurso sobre mi país, en Joseph Beuys, de Bernárdez (2003) donde expone su visión social del arte y afirma que cada hombre es un artista, postura que transfiere al contexto social con el concepto ampliado del arte. Lo anterior con el propósito de reflexionar sobre la naturaleza de los sistemas organizados y de cómo se potencia en el trabajo colectivo para la realización de una meta común, en este caso a través del arte, mediante la pintura de gran formato en equipos.

La sesión inicia con la Inducción, como fase de entrada, en donde se recibe a los participantes y se les facilita realizar la transición del afuera hacia el interior del espacio.

81 La Introducción tiene lugar una vez que los participantes centran la atención en el salón, se plantea el objetivo de la sesión, la reflexión general y se dan las orientaciones necesarias para el trabajo. La presentación de la sesión La conciencia de grupo, se basa en la propuesta de Joseph Beuys (2003), quien busca el bienestar humano a través de la comprensión de una nueva forma del trabajo, el cual establece dentro de un marco de creatividad y libertad dirigido al conjunto social, pues supone que se encuentra gravemente enfermo y no existen organismos que las garanticen. Este marco considera que cada hombre, es un artista, al desenvolverse él mismo como respuesta a su propia vocación: "La creatividad es únicamente lo que puede definirse y justificarse como ciencia de la libertad." (BERNARDÉZ S., C, 2003: p. 101). Esto lo lleva a promover la idea del arte como un universo interdisciplinar donde en conjunto se crea el concepto ampliado del arte. Asimismo, en (Bernardéz S. C. (2003), Beuys establece principios que se realizan en todos los niveles de la actividad humana, así como, en cada centro de trabajo, con el objeto de incidir en el sistema con una dinámica de proceso social, que concurra en el beneficio de la colectividad.

82 La fase de Desarrollo es a la práctica artística guiada, durante la cual mediante el consenso del equipo se crea una pintura de gran formato. 
83 Finalmente, la fase de Cierre de la sesión, procede cuando termina la práctica artística guiada, en donde se recomienda reorganizar el espacio para dejarlo como lo encontraron y concluir con los comentarios sobre la experiencia.

\section{Décima sesión: Compartir la experiencia}

La décima y última sesión Compartir la experiencia, se estructura sobre los fundamentos de Imber-Black, E., Roberts, J. y Whiting, R. (1997) en Rituales terapéuticos y ritos en la familia, para dar lugar al cierre por medio de la función del ritual, con el objetivo de crear un contexto favorable para la despedida y facilitar el cierre de los procesos del Taller de Creatividad, así como reflexionar y comprender los aprendizajes más significativos, mediante la escritura compartida de dichos aprendizajes y el montaje de una exposición colectiva.

La sesión inicia con la Inducción, esta corresponde a la fase de entrada, en donde se recibe a los participantes y se les facilita realizar la transición del afuera hacia el interior del espacio.

La Introducción inicia una vez que los participantes orientan la atención en el salón, se plantea el objetivo de la sesión, la reflexión general y se dan los lineamientos generales para el trabajo. La reflexión de la sesión Compartir la experiencia, se basa en la propuesta de Imber-Black, E., Roberts, J. y Whiting, R. (1997), quienes afirman que los rituales son actos simbólicos colectivos o individuales que facilitan la transición de un estadio a otro, los cuales incluyen rasgos ceremoniales y procesos de preparación. Estos están formados por partes abiertas y cerradas que se unen a través del acto simbólico como metáfora orientadora y pueden o no incluir palabras. De acuerdo con Myerhoff (1977) en Rituales terapéuticos y ritos en la familia, el ritual se distingue por representar la realidad en un espacio y tiempo sagrados. De tal suerte, agrega, que se puede establecer por un lado, como un sistema simbólico de intercomunicación entre un nivel de pensamiento y sus significados culturales y, por el otro, como un acto social y el hecho inmediato. Así, el ritual es fundamental dentro del orden social, pues tiene la función de transformar su estructura y establecer otras formas y tradiciones. En estos términos, el ritual no solamente orienta una transición, sino que también la realiza en un orden determinado.

87 La fase de Desarrollo corresponde a la práctica artística guiada, la cual consiste en dar lugar a que cada participante revise los trabajos realizados durante el Taller de Creatividad y escriba sobre los aprendizajes más significativos; al terminar se monta la exposición.

88 Finalmente, en la fase de Cierre de la sesión se invita a los padres, familiares y/o amigos a dar un recorrido donde se comparte la experiencia. 


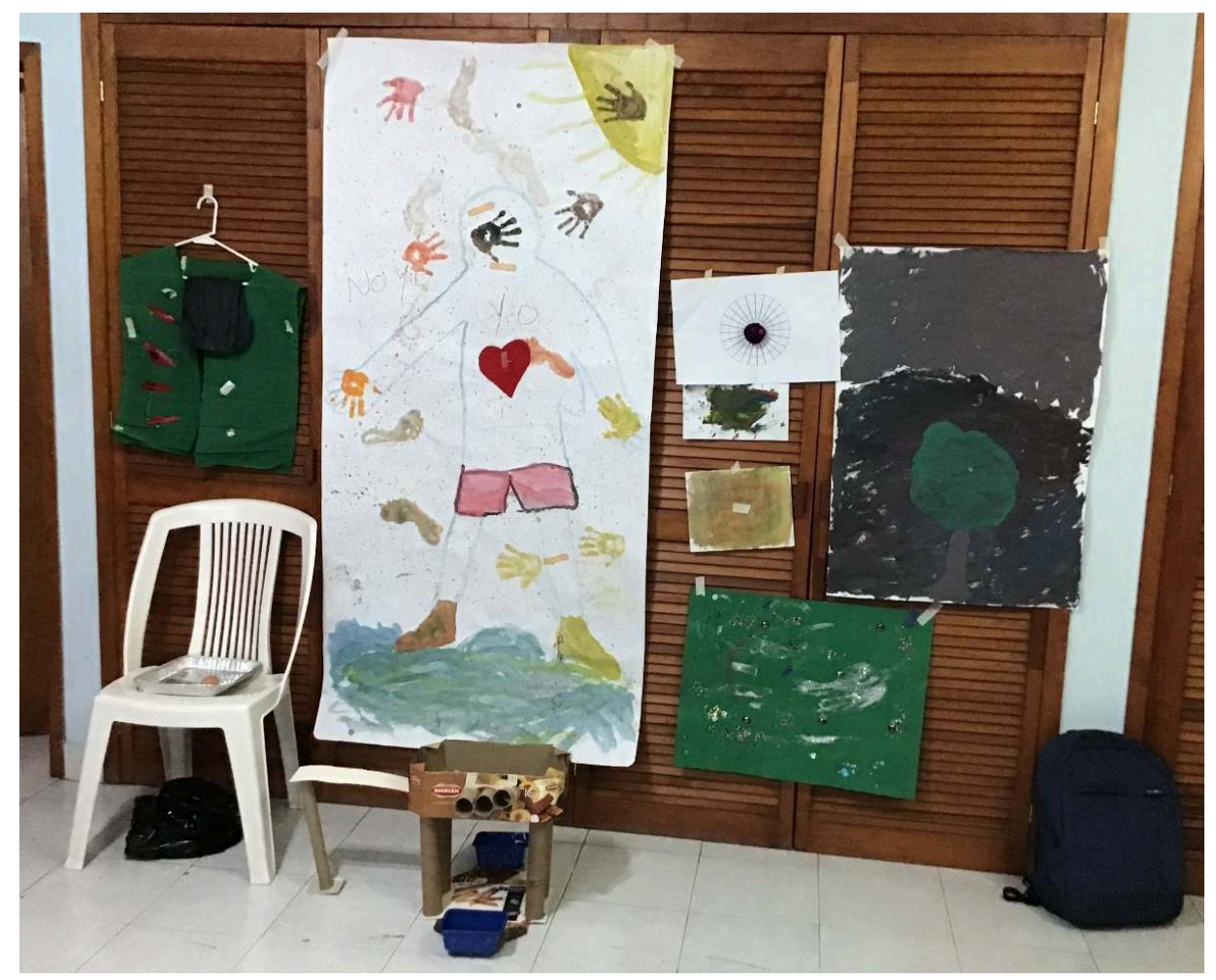

Imagen 5: Aguilar, G. (2020), Sesión 10: Compartir la experiencia

\section{Conclusiones} durante la experiencia de facilitación de los procesos creativos a través del Taller de Creatividad dan cuenta que todo ser humano es creativo por naturaleza, sin embargo, es necesario establecer determinadas condiciones para su desarrollo. Dichas circunstancias suponen la creación de un contexto diseñado tanto para la reflexión personal como espacio seguro, así como para la realización de la práctica artística guiada a través de los diferentes lenguajes artísticos, en donde ha sido posible establecer una metodología para diseñar el proceso creativo.

Esto es central, como se ha observado, la práctica artística guiada devuelve al sujeto a su condición simbólica, el cual es el único estado que permite la transformación de la subjetividad y los aprendizajes significativos, dando lugar a la expansión de la consciencia.

En este orden, la investigación concluye que los procesos de formación de la identidad y los procesos creativos se encuentran ligados profundamente y suceden de manera simultánea, de tal suerte que todo proceso de desarrollo de la identidad es un acto creativo y todo proceso creativo es un factor transformador de la personalidad, pues transitan por las mismas fases y poseen las mismas pautas que los definen. Finalmente, la respuesta a la pregunta inicial: ¿El Taller de Creatividad promueve la reflexión y el cambio en la postura subjetiva del sujeto a favor de una experiencia vital más satisfactoria? Es afirmativa puesto que los sujetos sanos nos encontramos en un permanente proceso de cambio, en la búsqueda que realiza el deseo para la obtención 
del placer, pues se trata de un: "[...] proceso subjetivante de restauración simbólica." (AGUILAR, 2016: p. 301)

Notas finales: La ilustración del texto con fotografías se ha visto limitada por motivos de espacio.

\section{BIBLIOGRAFÍA}

Aguilar, G. (2016), Procesos creativos y transformaciones subjetivas, La resignificación de la identidad a través de la experiencia artística dirigida, Tesis de Doctorado en Cultura y Arte, Universidad de Guanajuato, Campus León

Bernárdez Sanchís, C. (2003), Josep Beuys. Madrid: Nerea

Bertherat, T. (2002), El cuerpo tiene sus razones. México: Paidós

Foucault, M. (2010). Historia de la sexualidad: La inquietud de sí. México: Ediciones Gandhi

Freud, S. (1915). Pulsiones y destinos de pulsión. Buenos Aires: Obras completas. Colección digital Psicolibro. Vol. XIV.

Freud, S. (1923). El yo y el ello. Buenos Aires: Obras completas. Colección digital Psicolibro. Vol. XIX.

Imber-Black, E., Roberts, J. y Whiting, R. (1997). Rituales terapéuticos y ritos en la familia. Madrid: Gedisa

Laplanche, J. y.-B. (2004). Diccionaro de Psicoanálisis. México: Paidós.

Laplanche, J. (2002). La sublimación, Problemáticas III. Buenos Aires: Amorrortu

Lacan, J. (1954). El universo simbólico, Seminario 2, Clase 3. Buenos Aires: Obras completas. Colección digital Psicolibro.

Lacan, J. (1954). La tópica de lo imaginario, Seminario 1, Clase 7. Buenos Aires: Obras completas. Colección digital Psicolibro.

Robles, T. (2005). Manual de grupo de crecimiento. México: Alom.

Waisburd, G. y Sefchovich, G. (1999) en Expresión corporal y creatividad. México: Trillas

Waisburd, G. (2003). Creatividad y transformación. México: Trillas.

Zinker, J. (1997). El proceso creativo en la terapia gestáltica. México: Paidós.

\section{RESÚMENES}

La creatividad como cualidad humana, facilita la transformación de la subjetividad del sujeto, así como de su entorno, siempre y cuando existan las condiciones necesarias para su desarrollo. El presente texto tiene como propósito exponer el programa: Taller de Creatividad, con sus respectivas aproximaciones teórico-metodológicas, como dispositivo de reflexión personal para la resignificación de la experiencia vital a través de la creatividad artística. Esto es relevante, 
porque frecuentemente se observan problemáticas humanas asociadas al desdibujamiento de la identidad, influenciadas por la cultura actual, la cual, lejos de favorecer la toma de contacto con el yo auténtico para su significación, promueve la desconexión. En ese sentido, se encontró que la práctica artística guiada devuelve al sujeto a su condición simbólica.

La créativité comme qualité humaine, facilite la transformation de la subjectivité du sujet, ainsi que de son environnement, tant que les conditions nécessaires existent pour le développer. L'objectif de ce texte est d'exposer le programme: Atelier de Créativité, avec leurs approches théorico-méthodologiques respectives, comme dispositif de réflexion personnelle pour la résignation de l'expérience de vie par la créativité artistique. C'est pertinent, parce que les problèmes humains liés au brouillage identitaire sont fréquemment observés, influencé par la culture actuel, qui, loin de favoriser le contact avec le moi authentique pour sa signification, favorise leur déconnexion. Dans ce sens, on a constaté que la pratique artistique guidée ramène le sujet à sa condition symbolique.

\section{ÍNDICE}

Mots-clés: Créativité artistique - Condition symbolique - Pratique artistique guidée - Processus créatif - Atelier de créativité

Palabras claves: Creatividad artística - Condición simbólica - Práctica artística guiada - Proceso creativo - Taller de Creatividad

\section{AUTOR}

\section{GUADALUPE DE LA CRUZ AGUILAR SALMERÓN}

Profesora Investigadora - Departamento de Estudios Culturales - División de Ciencias Sociales y Humanidades - Universidad de Guanajuato, Campus León, México 